Europhysics Letters

PREPRINT

\title{
Off-equilibrium dynamics of the two-dimensional Coulomb glass
}

\author{
D. R. GRempel \\ CEA-Saclay, DSM/DRECAM/SPCSI, 91191 Gif-sur-Yvette, France
}

PACS. 75.10.Nr - Spin-glass and other random models.

PACS. 71.55. Jv - Disordered structures; amorphous and glassy solids.

PACS. 72.20.-i - Conductivity phenomena in semiconductors and insulators.

\begin{abstract}
The dynamics of the 2D Coulomb glass model is investigated by kinetic Monte Carlo simulation. An exponential divergence of the relaxation time signals a zero-temperature freezing transition. At low temperatures the dynamics of the system is glassy. The local charge correlations and the response to perturbations of the local potential show aging. The dynamics of formation of the Coulomb gap is slow and the density of states at the Fermi level decays in time as a power law. The relevance of these findings for recent transport experiments in Anderson-insulating films is pointed out.
\end{abstract}

A low-temperature glassy phase that had been anticipated by several authors $[1,2]$ was recently observed in studies of hopping conduction in disordered InO films [3,4]. The experiments show sluggish, non-exponential, relaxation of the conductance [3] as well as aging and memory effects [4] similar to those observed in glasses [5]. The details of the dependence of the observed effects on carrier density and sample conductance suggests that the glassiness of these systems is associated to the interplay of disorder and electron interactions [4].

The experimental systems, with very low carrier densities and very large resistances, are deep in the Anderson-Mott localized phase. In this regime quantum effects are qualitatively unimportant and a description based on the classical 2D Coulomb glass model is appropriate. The Hamiltonian of the model is [10]

$$
H=\sum_{i} n_{i} \varphi_{i}+\frac{e^{2}}{2 \kappa} \sum_{i \neq j} \frac{\left(n_{i}-K\right)\left(n_{j}-K\right)}{\left|\vec{r}_{i}-\vec{r}_{j}\right|} .
$$

Here, $\vec{r}_{i}$ denotes the position of the $i$-th localized state in the plane of the sample, $\varphi_{i}$ its energy, $e$ the electron charge and $\kappa$ the background dielectric constant. The electron occupancy $n_{i}=0,1$, and there is a uniform compensating background charge density $K=1 / N \sum_{i} n_{i}$ that ensures global charge neutrality. The presence of the long range Coulomb interaction in (11) is a salient feature of this model that has strong effects on its low-temperature properties.

The Coulomb glass model has been intensively investigated by Monte Carlo simulation but so far the main emphasis has been on its low-temperature properties at equilibrium $[1,6-9]$. In this Letter we report the results of the first systematic investigation of its off-equilibrium dynamics.

(c) EDP Sciences 
The time evolution of Anderson insulators takes place through phonon-assisted processes in which electrons hop from occupied sites $a$ to empty sites $b$ with a transition rate [10]

$$
\begin{aligned}
& \Gamma_{a \rightarrow b}=\tau_{0}^{-1} \exp \left(-\frac{2 R_{a b}}{\ell}\right) \Phi\left(\frac{\Delta E_{a b}}{T}\right), \\
& \Delta E_{a b}=\epsilon_{b}-\epsilon_{a}-\frac{e^{2}}{\kappa R_{a b}}, \quad \epsilon_{a}=\varphi_{a}+\frac{e^{2}}{\kappa} \sum_{b \neq a} \frac{\delta n_{b}}{R_{a b}} .
\end{aligned}
$$

Here, $\tau_{0}$ is a microscopic timescale, $\ell$ is the spatial extension of the localized wavefunctions, $\delta n_{a} \equiv n_{a}-K$ and $\Delta E_{a b}$ is the difference in energy between the final and initial states. The origin of the exponential factor in Eq. (2) is the dependence of the electron-phonon tunneling matrix element between sites $a$ and $b$ on the distance between them and $\Phi(x)$ is the thermal factor, $\Phi(x)=e^{-x}$ for $x>0, \Phi(x)=1$ for $x<0$. The positions $r_{i}$ of the localized states and their energies $\varphi_{i}$ are both random in general. However, we follow previous authors $[6,7]$ and set $\varphi_{i}=0$ thus limiting our discussion to a simplified model with positional disorder only $\left({ }^{1}\right)$. Furthermore, we take $K=1 / 2$ endowing the model with particle-hole symmetry, a feature that greatly simplifies the analysis of the results $[6,7]$. The substitution $2 n_{i}-1 \equiv \sigma_{i}= \pm 1$ makes Hamiltonian (1) formally equivalent to that of a classical disordered antiferromagnet with long range $1 / R$ interactions. The dynamics (2) would be quite unusual in the magnetic context, however $\left({ }^{2}\right)$.

We measure distances and energies in units of the average intersite distance $a_{0}$ and the Coulomb energy $E_{C}=e^{2} /\left(\kappa a_{0}\right)$ respectively, and we fix $\ell=a_{0}$. Using parameters appropriate for InO this corresponds to a carrier density $n \sim 3 \times 10^{21} \mathrm{~cm}^{-3}$ which is within the range of values studied experimentally [3,4]. For these values of the parameters $E_{C} \approx 0.1 \mathrm{eV}$ is huge compared to the experimental temperatures.

We performed kinetic Monte Carlo simulations of model (1). The localization centers are randomly distributed within a simulation cell of side $L$ containing $N_{e}=L^{2} / 2$ electrons. The cell is then periodically repeated in $2 \mathrm{D}$ space. The contribution to the potential within the simulation cell of charges located outside it is computed using the Ewald method. We start the simulations from a random electron configuration to mimic a quench from high temperature and then let the system evolve at the working temperature $T$ with the dynamics (2). The latter is implemented as follows. An occupied site $a$ is picked at random and a probability $P(b \mid a) \propto \exp \left(-2 R_{a b} / \ell\right)$ is assigned to each of the available empty sites $b$. A destination site is then picked from this probability distribution and an attempt is made to move the electron on site $a$ to $b$. The move is accepted with probability $\Phi\left(\Delta E_{a b} / T\right)$. A Monte Carlo step (MCS) consists of $N$ such attempts. We simulated systems with $L=8,16,32$ and 64 in the temperature range $0.01 \leq T \leq 0.1$. The length of our runs was typically $2 \times 10^{6}$ MCS. Physical quantities were monitored as a function of time and the results were averaged over between 64 and 6400 realizations of the disorder and the initial conditions, depending on size and temperature.

We begin our discussion by considering the local charge correlation function,

$$
C\left(t+t_{w}, t_{w}\right)=\frac{4}{N} \sum_{i}\left\langle\delta n_{i}\left(t+t_{w}\right) \delta n_{i}\left(t_{w}\right)\right\rangle,
$$

$\left.{ }^{1}\right)$ The symmetry breaking field $\varphi_{i}$ changes the universality class of the problem but the qualitative features of the off-equilibrium dynamics are preserved, at least if the random local potential is small enough. See also our concluding remarks

$\left({ }^{2}\right)$ Eq. (2) is a widely used approximate form for the transition rate in hopping-conduction systems. We expect other forms for $\Gamma_{a \rightarrow b}$ to lead to a qualitatively similar dynamics. 

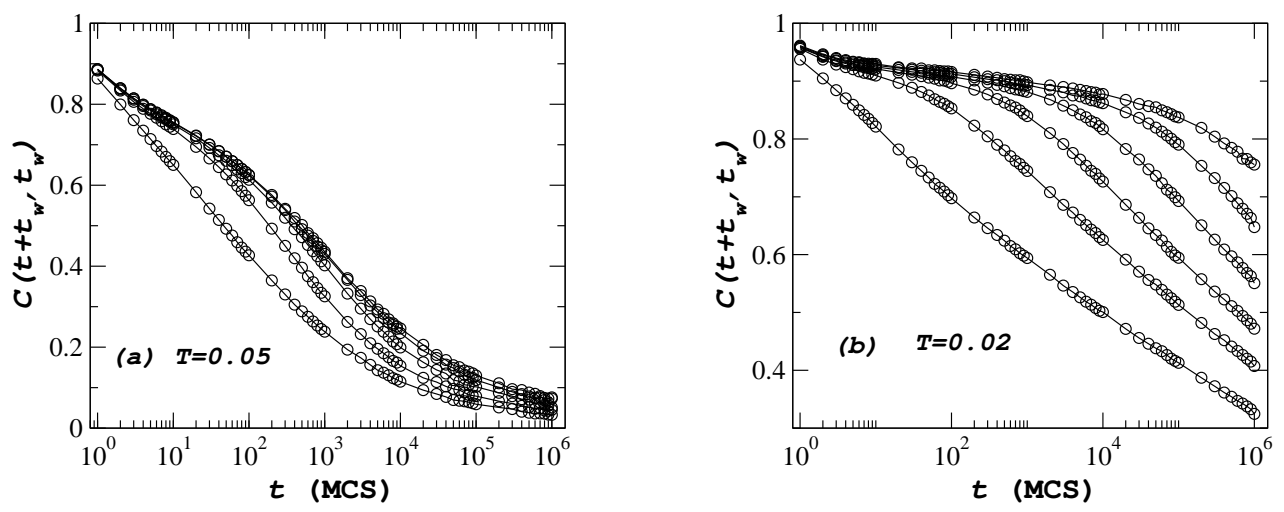

Fig. $1-C\left(t+t_{w}, t_{w}\right)$ for $t_{w}=10,10^{2}, 10^{3}, 10^{4}, 10^{5}$ and $10^{6}$, from bottom to top, for $T=0.05$ (a) and $T=0.02(\mathrm{~b})$.

where the brackets denote the average over configurational disorder and thermal noise and the waiting time $t_{w}$ is defined as the time elapsed since the quench. If $t_{w}$ is longer than the equilibration time $\tau_{e q}$ there is time-translation invariance and the correlation function will depend on the time difference $t$ only. Otherwise, $C$ is expected to depend on both $t$ and $t_{w}$.

The $T$-dependence of $\tau_{e q}$ was determined using the method of Bhatt and Young [11]. This is based on the comparison of two overlap functions, $Q_{r e p}\left(t_{0}\right)=\left\langle\left[4 / N \sum_{i} \delta n_{i}^{a}\left(t_{0}\right) \delta n_{i}^{b}\left(t_{0}\right)\right]^{2}\right\rangle$ and $Q_{t}\left(t_{0}\right)=\left\langle\left[4 / N \sum_{i} \delta n_{i}^{a}\left(2 t_{0}\right) \delta n_{i}^{a}\left(t_{0}\right)\right]^{2}\right\rangle$. In these expressions the superscripts $a$ and $b$ denote two copies of the system (i.e., two systems with the same realization of the disorder) that evolve independently starting from different uncorrelated initial conditions. If $t_{0} \geq \tau_{e q}$ the system is at equilibrium in which case [11] $Q_{r e p}\left(t_{0}\right)=Q_{t}\left(t_{0}\right)$. The equilibration time may thus be defined as the value of $t_{0}$ at which the two curves merge (within the statistical uncertainty). Fig. 2 shows the time-dependence of these overlap functions for a system of size $N=256$ and several temperatures. From a quantitative analysis of these results we find [cf. inset to the figure] that $\tau_{e q}(T) \sim \exp \left(T_{0} / T\right)$ where, restoring units, $T_{0} \sim E_{C} / 2$. The exponential divergence of $\tau_{e q}$ at $T=0$ is consistent with a freezing transition at $T_{g}=0$ as reported by other authors $[6,8]$. The equilibration time is thus finite for all $T \neq 0$ but it becomes very long for $T \ll E_{C}$. For $T>0.05$ our observation time is longer than $\tau_{e q}$ but the latter far exceeds the simulation time for $T<0.04$; in this $T$-range we can only probe the off-equilibrium dynamics. These temperature regimes are illustrated in Fig. 1 that shows the auto-correlation function for two temperatures, $T=0.05$ and $T=0.02$, and several values of $t_{w}$. At the higher temperature the relaxation curves for different values of $t_{w}$ coincide for $t_{w}>10^{5} \mathrm{MCS} \sim \tau_{e q}$ indicating that equilibrium was attained in the course of the simulation. At the lower temperature, however, the $t_{w}$-dependence persists throughout the observation time and the relaxation becomes increasingly sluggish as the system gets older. Equilibrium was obviously not reached within the simulation time in this case.

The size-dependence of our results in the non-stationary regime is remarkably weak as shown in Fig. B This is the signature of a very slow growth of the size of ordered domains $\xi(t)$ after a quench, a familiar feature of off-equilibrium glassy systems. An analysis of the four-point correlation function $\mathcal{C}^{(4)}\left(R_{i j}\right)=\left\langle\delta n_{i}^{a} \delta n_{i}^{b} n_{j}^{a} \delta n_{j}^{b}\right\rangle$ along the lines of Reference [12] gives access to $\xi(t)\left({ }^{3}\right)$. As illustrated in the inset to Fig. [3 at low temperature, $\xi(t) \propto \log t$

$\left({ }^{3}\right)$ Here we use the simple estimate $\xi^{2}(t)=\sum_{i, j} R_{i j}^{2} \mathcal{C}^{(4)}\left(R_{i j}\right) / \sum_{i, j} \mathcal{C}^{(4)}\left(R_{i j}\right)$. 


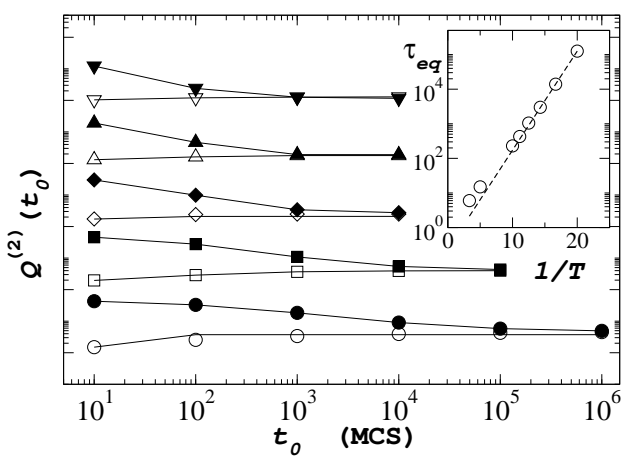

Fig. 2

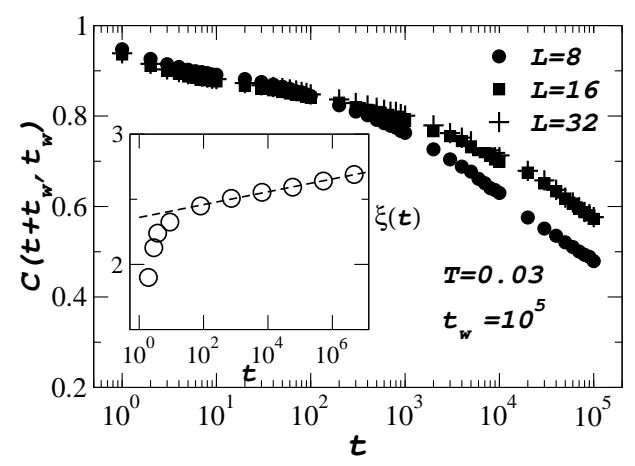

Fig. 3

Fig. 2 - The overlap functions $Q_{r e p}$ (open symbols) and $Q_{t}$ (filled symbols). The curves have been displaced vertically for the sake of clarity. The temperatures are $0.05,0.06,0.07,0.08$ and 0.09 , form bottom to top. Inset : the $T$-dependence of the equilibration time in MCS.

Fig. 3 - Size-dependence of $C\left(t+t_{w}, t_{w}\right)$ for $T=0.03, t_{w}=10^{5}$ and $L=8,16$ and 32 . Inset: timedependence of the domain size $\xi$ determined from the analysis of the four point correlation function at $T=0.03$.

reaches only a few lattice spacings in the course of our simulations.

Let us discuss in more detail the off-equilibrium regime illustrated by the data of Fig. 10 (b). At equilibrium, the correlation function has the scaling form $C(t) \sim t^{-\lambda} \mathcal{C}_{\text {eq }}(t / \tau)$ with $T$ dependent $\lambda$ and $\tau$. In the non-stationary regime this expression generalizes to $[12,13]$

$$
C\left(t+t_{w}, t_{w}\right) \sim t^{-\lambda} \mathcal{C}_{\mathrm{ag}}\left(\frac{h\left(t+t_{w}\right)}{h\left(t_{w}\right)}\right) .
$$

The above form for the argument of the scaling function $\mathcal{C}_{\text {ag }}$, often used in discussions of experimental data in glasses [5], also arises in the exact solution of certain mean-field models [14]. Here we adopt the widely used parameterization [5] $h(x)=\exp \left[x^{1-\mu} /(1-\mu)\right]$. In the limit $\mu \rightarrow 1$ this expression leads to simple aging, $C\left(t+t_{w}, t_{w}\right) \sim t^{-\lambda} \mathcal{C}_{\text {ag }}\left(t / t_{w}\right)$; for other values of $\mu$ it leads to an effective relaxation increasing with age as $t_{w}^{\mu}$. We used Eq. (5) to perform a scaling analysis of the data of Fig. 1 (b) as well as those obtained for two other temperatures, $T=0.01$ and $T=0.03$. The results are displayed in Fig. (4) We see that Eq. (5) leads to an excellent collapse of the scaled data for the three temperatures. The exponent $\lambda$, small in magnitude, varies substantially with temperature. The values obtained are consistent with $\lim _{T \rightarrow 0} \lambda(T)=0$ as expected for system that freezes at $T=0$. The variation of the parameter $\mu$ with $T$ is much less pronounced. We find simple aging $(\mu \rightarrow 1)$ as $T \rightarrow 0$ and a slight tendency to sub-aging with increasing $T$. It is worth noticing that simple aging was observed in the conductivity of Anderson-insulating films [4].

It has been recently suggested [15] that some of the experimentally observed features may be due to a slow response of the system to the changes in the local random potential $\varphi_{i}$ [cf. Eq. (10]. We thus studied the response of the system to random perturbations of the form $\delta \varphi_{i}=\epsilon_{i} \varphi_{0}$ where $\varphi_{0} \ll 1$ is the overall scale of the perturbation and $\epsilon_{i}$ are normalized random variables uncorrelated from site to site, $\left\langle\epsilon_{i} \epsilon_{j}\right\rangle=\delta_{i j}$. This perturbation is switched on at time $t_{w}$ and its effect on the system is observed a time $t$ later. The quantity conjugated to the random potential is $\delta n(t) \equiv 1 / N \sum_{i}\left\langle\delta n_{i}(t) \epsilon_{i}\right\rangle$. The results of our measurements of the response of the system are shown in Fig. (5) for the case of a random potential of amplitude 


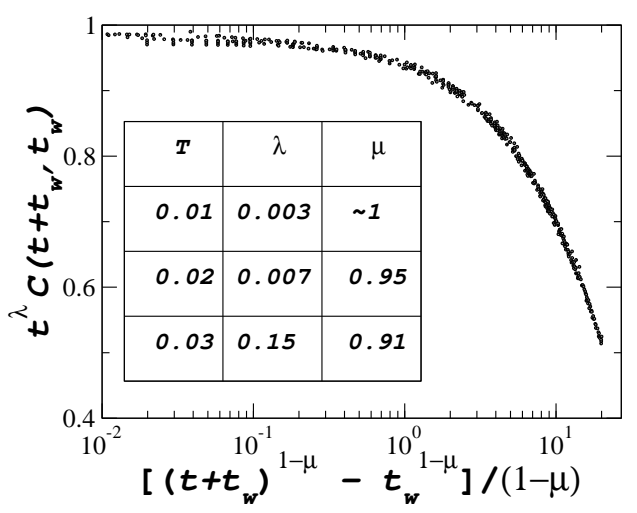

Fig. 4

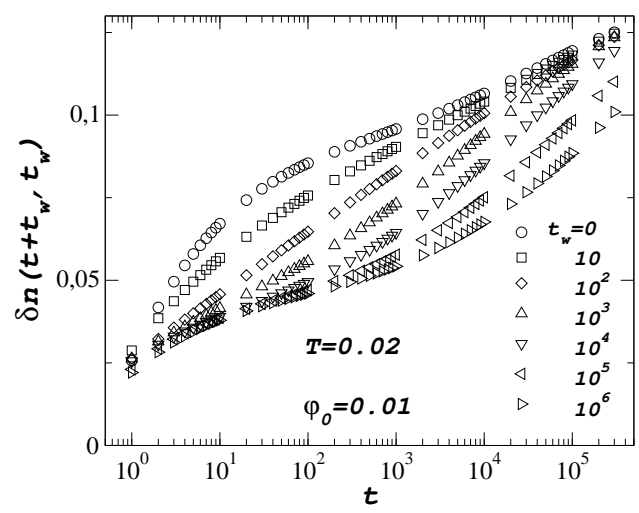

Fig. 5

Fig. 4 - Scaling plot of $C\left(t+t_{w}, t_{w}\right)$. The symbols represent the data obtained for three temperatures, $T=0.01,0.02$ and 0.03 . Inset: the $T$-dependence of the exponents $\mu$ and $\lambda$.

Fig. 5 - Response to a random local field of amplitude $\varphi_{0}=0.01$ for $T=0.02$.

$\varphi_{0}=0.01$ and $T=0.02$. It may be seen that the response $\delta n$ exhibits aging just as the correlation function $C$ does. As $t_{w}$ increases, the response gets more and more sluggish and, within the time window explored, $\delta n(t) \approx \ln t$ for $t \gg t_{w}$.

It can be easily shown that, in the linear response regime,

$$
\delta n(t)=\varphi_{0} \chi\left(t+t_{w}, t_{w}\right), \quad \chi\left(t, t^{\prime}\right)=\int_{t^{\prime}}^{t} d t^{\prime \prime} R\left(t, t^{\prime \prime}\right),
$$

where $\chi$ is the local susceptibility and $R$ is the local response function. At equilibrium, $\chi$ and $C$ are related by the fluctuation dissipation theorem (FDT), $T \chi(t)=1-C(t)$. We show in Fig. 6] (a) a parametric plot of the product $T \chi$ as a function of $C$ for $T=0.02$ and several waiting times. It can be seen that our data violate this relationship: whereas for each $t_{w}$ the points do align on the FDT straight line for short times $(C \sim 1)$, they deviate from it at long times (small $C$ ). The value of $C$ at which deviations first appear slowly increases with the age of the system.

A generalization of FDT was found to hold for many model systems [14] in which, for long $t$ and $t^{\prime}$, the off-equilibrium $\chi\left(t, t^{\prime}\right)$ still depends on the times only through its dependence on $C, \chi\left(t, t^{\prime}\right)=\Theta\left[C\left(t, t^{\prime}\right)\right]$ where $\Theta$ is in general non linear. In glassy systems below the glass transition the FDT curves for different values of $t_{w}$ converge to the limiting $\Theta[C]$ as $t_{w} \rightarrow \infty$. Our data are compatible with $\Theta[C]$ consisting of two straight lines with different slopes with a break point at some value $C^{\star}$ of the correlation. This type of behavior behavior is found in some mean-field spin-glass models [14] and in models of structural glasses [17] but not in short-range spin glasses. Since our two-dimensional model has no finite-temperature glass transition, we should see the break point continuously shift to lower values of $C$ with increasing $t_{w}$ such that for $t_{w}>\tau_{e q}$ the classical FDT line is recovered. This process is slow, however, and our data only show a hint of it: on the time scale of the simulation the system behaves as a glass that violates FDT. We display in Fig. 6 (b) the temperature-dependence of the FDT diagram. It is seen that the break point gradually shifts to longer time scales with increasing $T$ until, by the time we reach $T=0.08$, FDT is fully restored.

In the study of models of glasses it has proven conceptually useful to introduce an effective temperature [16] $1 / T_{\text {eff }}=-\partial \Theta / \partial C$ that depends on timescale and represents (loosely 

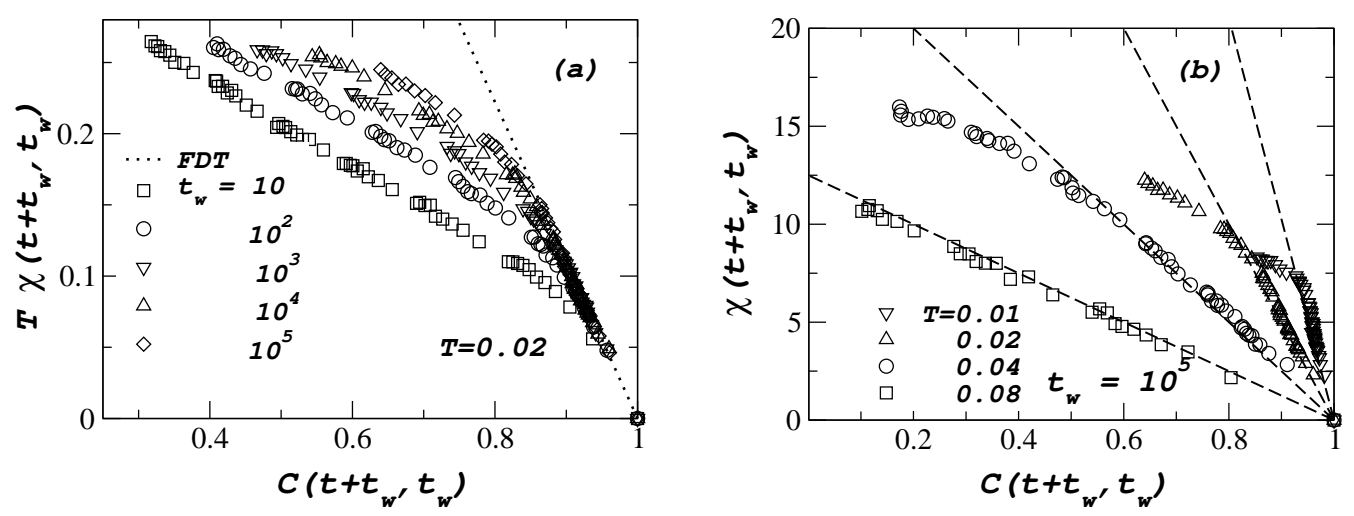

Fig. 6 - (a) Parametric plot of susceptibility vs. correlation for $T=0.02$ and the waiting times indicated in the legend. (b) $\chi$ vs. $C$ for $t_{w}=10^{5}$ and the values of $T$ given in the legend. The straight lines have slopes $1 / T$. FDT holds for the highest temperature. $T_{\text {eff }} \sim 0.08$ in this $T$-range.

speaking) the temperature of the un-equilibrated degrees of freedom. The data displayed in Fig. [6 (b) suggest that at low $T$, in the time-domain explored, there exist just two timescales, with effective temperatures $T_{\text {eff }}=T$ and $T_{\text {eff }} \sim 0.08$, respectively.

Slow relaxation can also be seen in other physical quantities such as the density of states $\rho(\epsilon)=1 / N\left\langle\sum_{i} \delta\left(\epsilon-\epsilon_{i}\right)\right\rangle$. It was suggested [18] that the slow relaxation of the conductance observed experimentally may reflect a slow formation of the Efros-Shklovskii Coulomb gap [10]. We display in Fig. (7) our results for $\rho(\epsilon)$ for a system of size $N=2500$ at $T=0.02$. It is seen that the density of states, featureless right after the quench, slowly develops a pseudo gap for $|\epsilon| \rightarrow 0$. The inset to the figure shows the time dependence of the density of states at the Fermi level, $\rho(0, t) \sim t^{-\nu}$ with $\nu \approx 0.3$. A power-law decay of $\rho(0, t)$ was predicted by the phenomenological approach of Reference [18].

The glassy behavior described above only exists on timescales shorter than $\tau_{e q}$. At fixed $T$, this increases exponentially with the Coulomb energy which is determined by the doping, $E_{C} \propto n^{1 / 3}$. For a carrier density $n=10^{21} \mathrm{~cm}^{-3}, E_{C} \sim 700 \mathrm{~K}$ which leads to values of $\tau_{e q}$ that are much greater than experimental timescales at the working temperature. The relaxation

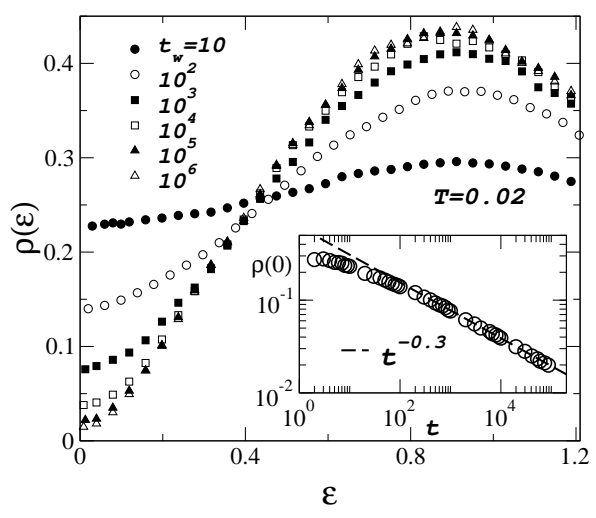

Fig. 7 - Time evolution of the Coulomb gap for $T=0.02$ for the waiting times shown in the legend. The size of the system is $N=2500$. Inset: power-law decay of the density of states at the Fermi level. 
time decreases very rapidly with decreasing $n$ implying an absence of glassiness in the more lightly doped samples, a trend that was observed in the experiments [4]. It is interesting to notice that 2D spin-glass films, which like the 2D Coulomb glasses discussed here do not have a finite temperature phase transition, also exhibit aging effects [19].

In summary, we studied the dynamics of the 2D Coulomb-glass model at low temperature. The equilibration time of the system diverges exponentially for $T \rightarrow 0$ signaling a transition at $T_{\mathrm{g}}=0$. At finite $T$, the evolution of the system after a sudden quench from high temperatures is similar to that of glasses below $T_{g}$ for times shorter than $\tau_{\text {eq }}$. In this regime the charge autocorrelation function and the response of to local random perturbations show simple aging and FDT violations are observed. The density of states close to the Fermi level evolves slowly and $\rho(0)$ has a power-law decay.

Our results (except those for the response function) were obtained in the absence of the symmetry breaking field $\varphi_{i}$. We checked explicitly that the aging behavior of $C\left(t, t^{\prime}\right)$ persists in the presence of a small random local potential $\left(\varphi_{0} \ll 1\right)$. We have preliminary evidence that the model with $\varphi_{0} \sim 1$ also ages, in contradiction with recent claims in the literature [9].

I thank Z. Ovadyahu, A. Vaknin, M. Pollak, L. F. Cugliandolo, A. Kolton and D. Dominguez for illuminating discussions. This work was supported in part by NSF Grant No. PHY9907949 and by the program ECOS-Sud, project A01E01.

\section{REFERENCES}

[1] Davies J. H. et al., Phys. Rev. Lett., 49 (1982) 758; ibid., Phys. Rev. B, 29 (1984) 4260.

[2] Pollak M., Philos. Mag. B, 50 (1984) 265; Grünewald M. et al., J. Phys. C, 15 (1982) L1153.

[3] Ben-Chorin M., et al., Phys. Rev. B, 48 (1993) 15025 ; Ovadyahu Z. and Pollak M., Phys. Rev. Lett.,79 (1997) 459.

[4] Vaknin A. et al., Phys. Rev. Lett., 84 (2000) 3402; ibid., Phys. Rev. B, 65 (2002) 134208; ibid, Phys. Rev. Lett., 81 (1998) 669.

[5] Struik L. C. E., Physical Aging in Amorphous Polymers and Other Materials (Elsevier, Amsterdam,1978); Vincent E. et al, in Complex behavior of Glassy Systems, M. Rubi and C. PerezVicente Eds. (Springer, Berlin, 1997).

[6] Xue W. and Lee P. A., Phys. Rev. B, 38 (1988) 9093.

[7] Grannan E. R. and Yu Clare C., Phys. Rev. Lett., 71 (1993) 3335.

[8] Menashe D. et al., Europhys. Lett., 52 (2000) 94; ibid., Phys. Rev. B, 64 (2001) 115209.

[9] Tsigankov and Efros A. L., Phys. Rev. Lett., 88 (2002) 176602 ; Tsigankof D. N. et al., Phys. Rev. B, 68 (2003) 184205.

[10] Electron-Electron interactions in Disorder Systems, A. L. Efros and M. Pollak, Editors (NorthHolland, Amsterdam, 1985).

[11] Bhatt R. N. and Young A. P., Phys. Rev. Lett., 54 (1984) 924.

[12] Kisker J. et al., Phys. Rev.B, 53 (1996) 6418; Berthier L. and Bouchaud J.-P., Phys. Rev. B, 66 (2002) 054404.

[13] Godrèche C. and Luck J. M., J. Phys. A, 33 (2000) 9141.

[14] Cugliandolo L. F. and Kurchan J., Phys. Rev. Lett., 71 (1993) 173; ibid, J. Phys. A, 27 (1994) 5749.

[15] Vaknin A. et al., Phys. Rev. B, 61 (2000) 6692; ibid, Springer Proceedings in Physics, 87 (2001) 995.

[16] Cugliandolo L. F., Kurchan J. and Peliti L., Phys. Rev. E, 55 (1997) 3898.

[17] Parisi G., Phys. Rev. Lett., 79 (1997) 3660.

[18] Yu Clare C., Phys. Rev. Lett., 82 (1999) 4074.

[19] Schins A. G. et al., Phys. Rev. B, 48 (1993) 16524. 